\title{
Acute brainstem encephalitis associated with Mycoplasma pneumoniae in an adult: a case report
}

\author{
Min-Hee Woo, Jung-Won Shin \\ Department of Neurology, CHA Bundang Medical Center, CHA University, Seongnam, Korea
}

\begin{abstract}
Brainstem encephalitis (BE) associated with Mycoplasma pneumoniae in adults is rare, and the diagnosis is challenging. We describe an uncommon case of BE in an immunocompetent patient. A 43-year-old, otherwise healthy woman visited our emergency department with high fever and a sore throat, and 3 days later she returned with an altered drowsy mental status. Magnetic resonance imaging displayed diffuse swelling in bilateral cerebral regions involving the bilateral pons. The sera tested positive for the immunoglobulin (Ig) M antibody against M. Pneumoniae as detected by an enzyme immunoassay (EIA), and on hospital day 10, the level of IgM index against M. pneumoniae further increased from 1.5 to 2.1. We changed the antibiotic regimen from vancomycin and ceftriaxone to clarithromycin based on detection of M. pneumoniae, and we added intravenous immunoglobulin. After one month, the patient fully recovered from the neurological deficits. A follow-up brain magnetic resonance imaging was performed, which showed completely resolved lesions. Particle agglutination assay (PA) and EIA are both largely used to diagnose M. pneumoniae. Compared to the PA test, the EIA test could be a reliable tool because it separately measures IgM and IgG antibodies. We diagnosed BE associated with M. pneumoniae through EIA with an increasing level of IgM in the acute and subacute paired sera. Early treatment with macrolide antibiotics resulted in a good outcome.
\end{abstract}

Keywords: Encephalitis, Mycoplasma pneumoniae, Immunoenzyme techniques, Routine diagnostic tests

\section{Introduction}

The etiologies of brainstem encephalitis (BE) can be divided into infectious and autoimmune. The most common infectious causes are Listeria, enteroviruses, and herpes viruses [1]. Among these causes, central nervous system (CNS) infection with Mycoplasma pneumoniae is rarer in adult than in pediatric patients. CNS manifestations related to infection with $M$. pneumoniae are variable, and a positive serological result in an adult may simply reflect carriage or previous encounters with M. pneumoniae, making the diagnosis of $\mathrm{BE}$ associated with $M$. pneumonia particularly challenging in adult patients [2].

We report a case of $\mathrm{BE}$ associated with $M$. pneumoniae in an immunocompetent adult patient who was diagnosed based on an increased level of specific immunoglobulin (Ig) M anti- body against $M$. pneumoniae through enzyme immunoassays (EIAs) after we compared levels between the acute phase and the convalescent sera. The patient recovered without any sequelae despite initial severe neurological deficits.

\section{Case Report}

A 43-year-old, otherwise healthy woman visited our emergency department with high fever and a sore throat. She was discharged after symptom control, but 3 days later she returned with an altered, drowsy mental status (Glasgow coma scale, 4). Her body temperature was $38^{\circ} \mathrm{C}$, and the initial laboratory tests showed mild leukocytosis with slightly elevated C-reactive protein. A lumbar puncture was performed, and cerebrospinal fluid (CSF) analysis showed minimal pleocytosis (white

Received: July 14, 2021 Revised: September 27, 2021 Accepted: September 27, 2021

Correspondence: Jung-Won Shin

Department of Neurology, CHA Bundang Medical Center, CHA University, 59 Yatap-ro, Bundang-gu, Seongnam 13496, Korea

E-mail: limitsum@gmail.com

ORCID: https://orcid.org/0000-0003-2155-9068

Copyright (C) 2021 by The Korean Encephalitis and Neuroinflammation Society

This is an open access article distributed under the terms of the Creative Commons Attribution Non-Commercial License (http://creativecommons.org/licenses/by-nc/4.0/) which permits unrestricted non-commercial use, distribution, and reproduction in any medium, provided the original work is properly cited. 
blood cells, 3 cells $/ \mathrm{mm}^{3}$ ) with a slightly elevated protein concentration of $46 \mathrm{mg} / \mathrm{dL}$. The brain magnetic resonance imaging (MRI; T2-weighted and fluid-attenuated inversion recovery) displayed high signal intensities in the bilateral medial temporal cortices and posterior pons, more prominent in the left than the right (Figure. 1). We immediately administered intravenous (IV) ceftriaxone, vancomycin, acyclovir, and dexamethasone. On hospital day 3, IVIg was also administered since autoimmune encephalitis could not be ruled out. On hospital day 4, the patient's mental status recovered, but the patient complained of diplopia. The specific serological IgM test via EIA for M.pneumoniae was positive (1.5), and IgG was not detected. IgM measured by Chorus Trio instruments (DIESSE Diagnostic, Monteriggioni, Italy). The Chorus instrument expresses the result as an index (ratio between the optical density value of the test sample and that of the cutoff) which can be used as a quantitative measure, as it is proportional to the amount of specific IgM present. The test serum can be interpreted as follows: positive, when the ratio is $>1.1$; negative, when the ratio is $<0.9$; equivocal, for all values between 0.9 and 1.1. The results of IgG antibodies were expressed in $\mathrm{AU} / \mathrm{mL}$. To exclude extrapulmonary manifestations due to $M$. pneumoniae and other infectious conditions, we conducted echocardiography, neck sonography, and chest and abdominal pelvic computed tomography with contrast. The above examinations disclosed no abnormal findings. The antibiotic regimen was changed to clarithromycin due to infection with $M$. pneumoniae. Routine CSF Gram stain, bacterial cultures, and polymerase chain reaction (PCR) tests showed negative results for herpes simplex, varicella zoster, cytomegalovirus, Epstein-Barr virus, Mycobacterium, and $M$. pneumoniae. Tests for autoimmune antibodies (anti- $N$-meth-

Figure 1. T2-weighted and fluid-attenuated inversion recovery axial images
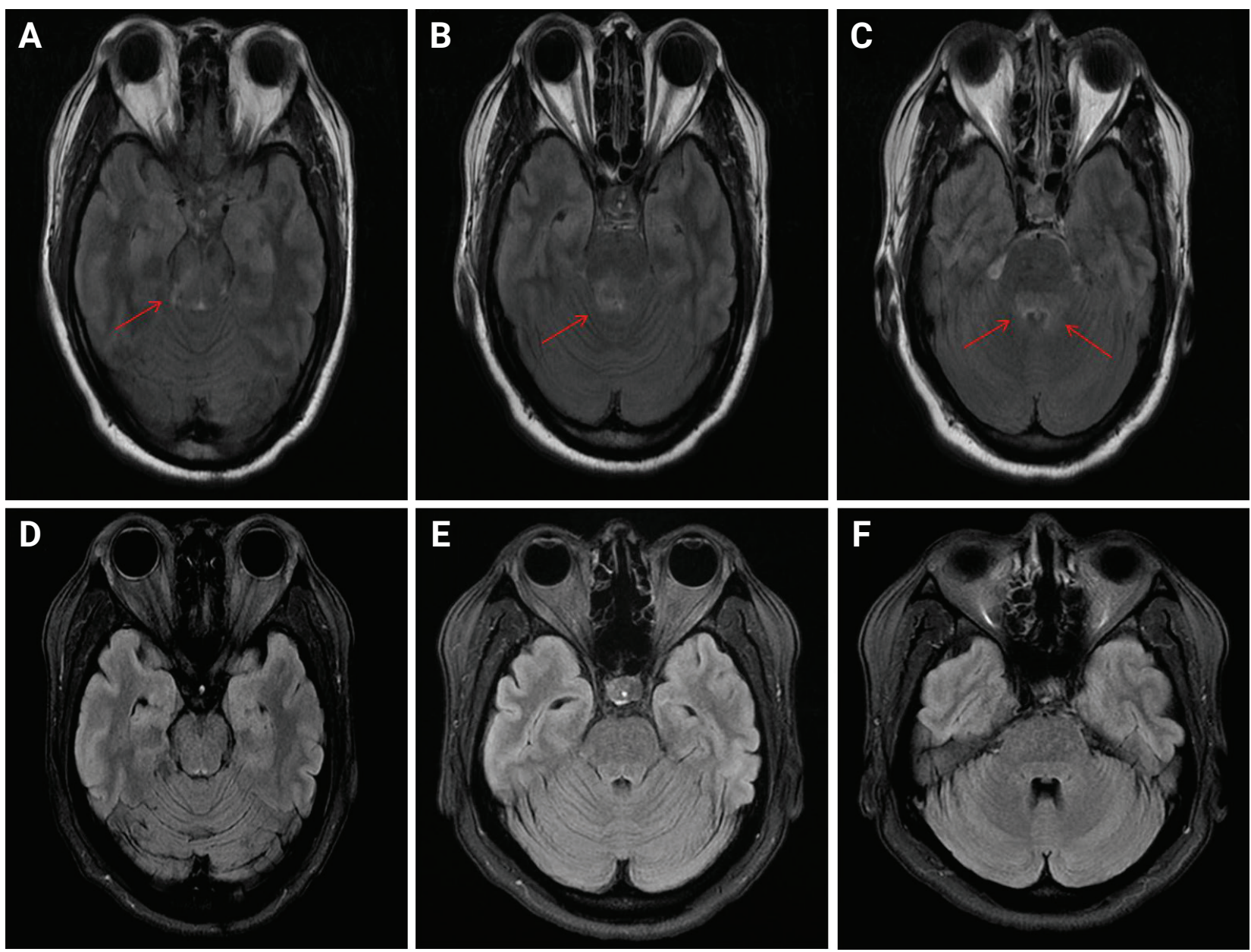

T2-weighted and fluid-attenuated inversion recovery axial images demonstrating diffuse swelling (red arrows) during the bilateral cerebral swelling that involved the bilateral pons at admission (A, B, and $\mathbf{C}$ ) and improvement 1 month after treatment (D, E, and F). 
yl-D-aspartate receptor [NMDAR], -LGI1, -CASPR2, -AMPA1, -AMPA2, -GABAB-R, -Hu, -Yo, -Ri, -Ma2, -CV2/CRMP5, and -amphiphysin) in the CSF and serum were negative. Additionally, serum anti GQ1b, GM1, and GD1b IgM antibodies were negative. On hospital day 10, the serum IgM value for $M$. pneumoniae increased to 2.1, and the IgG test remained negative. On hospital day 14, the patient's symptoms of diplopia and ataxic gait further improved. Therefore, we did not consider other immunotherapies, and antibiotic treatment was used for a total of 2 weeks. After 1 month, the patient fully recovered from the neurological deficits, a follow-up brain MRI was performed, which showed completely resolved lesions (Figure 1).

Written informed consent was obtained from the patient for publication of the case and any accompanying images.

\section{Discussion}

In this case report, we presented a middle-aged adult patient with BE associated with M. pneumoniae infection who was diagnosed via positive IgM tests in which increased levels were demonstrated in acute and convalescent sera with an EIA. The patient was successfully treated with clarithromycin and IVIg.

M. pneumonia is a well-known cause of respiratory tract disease, and extrapulmonary manifestations are also common [3]. It is frequently associated with encephalitis, especially in children. Lerer and Kalavsky [4] found that $53 \%$ of patients with CNS disease associated with $M$. pneumoniae were aged between 6 and 20 years. Only a few cases have been reported in adults above middle age, and one of these patients received an immunosuppressive agent [5-7]. Encephalitis caused by $M$. pneumoniae cannot be reliably diagnosed in adults for the following reasons; the incidence rate is low, no clinical or radiologic signs which could indicate a mycoplasma etiology of CNS disease exist, and a clear diagnostic marker in the CSF is absent. The detection rate of $M$. pneumoniae by PCR test in the CSF of patients with $M$. pneumoniae encephalitis is relatively low (0\%-14\%), and serologic tests are indispensable [2]. In addition, due to the high prevalence of $M$. pneumoniae infection, one measurement of high serum antibody titers may simply indicate carriage or a previous infection [8]. On the other hand, a reliable diagnosis may be achieved by using paired patient sera to detect seroconversion and/or an increase in antibody titers. The gold standard for diagnosis is a four-fold increased Ig titer in particle agglutination assays (PA) or complement fixation tests [2]. An EIA test can separately detect IgG and IgM to distinguish current from past infections. Several commercial EIA kits are now available, and in some studies, the EIA method had moderate-to-high sensitivity and specificity [2]. Furthermore, correlation of M. pneumoniae IgM values obtained by various IgM assays with PA titers was also noted [9]. In our case, the level of IgM was increased in paired sera, and the diagnostic value of an increasing titer should be confirmed in additional validation studies.

The pathogenesis of encephalitis associated with the respiratory pathogen $M$. pneumoniae is not well understood. A direct infection of the CNS and an immune-mediated process have been previously discussed [2]. Given several cases of Guillain-Barré syndrome [10], Bickerstaff BE [11], and NMDAR associated with Mycoplasma spp. infection[12], the immune response to $M$. pneumoniae in the CNS can be an important factor that contributes to encephalitis [6]. Therefore, the development of new diagnostic tools, such as those which detect antibodies against $M$. pneumoniae in the CSF, are necessary.

M. pneumoniae lacks a cell wall, and beta-lactate antibiotics are not suitable to treat the infection. Doxycycline, a macrolide, can be used, and corticosteroids may be beneficial according to a recent review of severe cases [2].

We diagnosed BE associated $M$. pneumoniae infection through EIA with an increasing titer of IgM in acute and subacute paired sera. Early treatment with macrolide antibiotics resulted in a good outcome for our patient. Additionally, IVIg was administrated for 5 days and might have supported her recovery. Immunomodulators, such as corticosteroids or Igs, should be beneficial for the most severe case, including encephalitis [13]. Considering the pathogenesis of immune-mediated processes, further studies on the efficacy of immunomodulatory treatment are necessary.

\section{Conflicts of Interest}

No potential conflict of interest relevant to this article was reported.

\section{Author Contributions}

Conceptualization: Shin JW; Data curation and Writing-original draft: Woo MH; Writing-Review and editing: all authors 


\section{References}

1. Jubelt B, Mihai C, Li TM, Veerapaneni P. Rhombencephalitis / brainstem encephalitis. Curr Neurol Neurosci Rep 2011;11:543-552.

2. Meyer Sauteur PM, Jacobs BC, Spuesens EB, et al. Antibody responses to Mycoplasma pneumoniae: role in pathogenesis and diagnosis of encephalitis? PLoS Pathog 2014;10:e1003983.

3. de Groot RC, Sauteur PMM, Unger WW, van Rossum AM. Things that could be Mycoplasma pneumoniae. J Infect 2017;74:S95-S100.

4. Lerer RJ, Kalavsky SM. Central nervous system disease associated with Mycoplasma pneumoniae infection: report of five cases and review of the literature. Pediatrics 1973;52:658-668.

5. Sugeno N, Kawaguchi N, Hasegawa T, et al. A case with anti-galactocerebroside antibody-positive Mycoplasma pneumoniae meningoencephalitis presenting secondary hypersomnia. Neurol Sci 2012; 33:1473-1476.

6. Christie LJ, Honarmand S, Yagi S, Ruiz S, Glaser CA. Anti-galactocerebroside testing in Mycoplasma pneumoniae-associated encephalitis. J Neuroimmunol 2007;189:129-131.

7. Lanczik O, Lecei O, Schwarz S, Hennerici M. Mycoplasma pneumoniae infection as a treatable cause of brainstem encephalitis. Arch Neu- rol 2003;60:1813-1813.

8. Daxboeck F. Mycoplasma pneumoniae central nervous system infections. Curr Opin Neurol 2006;19:374-378.

9. Yoo SJ, Oh HJ, Shin BM. Evaluation of four commercial IgG- and IgM-specific enzyme immunoassays for detecting Mycoplasma pneumoniae antibody: comparison with particle agglutination assay. J Korean Med Sci 2007;22:795-801.

10. Ang C, Tio-Gillen A, Groen J, et al. Cross-reactive anti-galactocerebroside antibodies and Mycoplasma pneumoniae infections in GuillainBarré syndrome. J Neuroimmunol 2002;130:179-183.

11. Steer AC, Starr M, Kornberg AJ. Bickerstaff brainstem encephalitis associated with Mycoplasma pneumoniae infection. J Child Neurol 2006;21:533-534.

12. Granerod J, Ambrose HE, Davies NW, et al. Causes of encephalitis and differences in their clinical presentations in England: a multicentre, population-based prospective study. Lancet Infect Dis 2010;10:835844.

13. Narita M. Classification of extrapulmonary manifestations due to Mycoplasma pneumoniae infection on the basis of possible pathogenesis. Front Microbiol 2016;7:23. 